\title{
An Adaptive Backoff Mechanism for IEEE 802.15.4 Beacon-Enabled Wireless Body Area Networks
}

\author{
Shagufta Henna (iD) and Muhammad Awais Sarwar \\ Department of Computer Science, Bahria University, Islamabad, Pakistan \\ Correspondence should be addressed to Shagufta Henna; shinna.buic@bahria.edu.pk
}

Received 14 March 2018; Revised 12 May 2018; Accepted 3 June 2018; Published 26 June 2018

Academic Editor: Manuel Fernandez-Veiga

Copyright ( 2018 Shagufta Henna and Muhammad Awais Sarwar. This is an open access article distributed under the Creative Commons Attribution License, which permits unrestricted use, distribution, and reproduction in any medium, provided the original work is properly cited.

\begin{abstract}
Carrier sense multiple access mechanism with collision avoidance (CSMA/CA) in IEEE 802.15.4-based wireless body area networks (WBANs) may impair the transmission reliability of emergency traffic under high traffic loads, which may result in loss of high valued medical information. Majority of the recent proposals recommend an early retransmission of failed frame while ignoring the history of past failed transmissions. More importantly, these proposals do not consider the number of failed transmissions experienced by each sensor node, thereby affecting the reliability of retransmissions. In this paper, we propose a dynamic retransmission adaptive intelligent MAC (RAI-MAC) scheme. In our proposed scheme retransmission class of each sensor node is decided by the coordinator according to the number of failed transmissions of each node as observed by the coordinator during the last superframe. Based on the retransmission class received from the coordinator, each node adjusts its next backoff value. The proposed scheme increases the probability of successful frame retransmissions without incurring extra overhead. The simulation results prove that the proposed scheme based on its adaptive retransmission mechanism achieves higher average throughput and average end-to-end delay, while not compromising on energy efficiency as compared to the IEEE 802.15.4 and Block Acknowledgment (Block Ack). Moreover, our scheme appears more stable in terms of average throughput, end-to-end delay, and energy efficiency under different values of beacon order (BO) and superframe order (SO).
\end{abstract}

\section{Introduction}

Recently, WBAN has emerged as a subcategory of wireless sensor networks (WSNs), which primarily aims to monitor physiological and ambient conditions of human beings and animals and report them to a coordinator [1]. These costeffective and low-duty cycle WBANs aim to provide reliable operation with low data rates, mobility support, and security with minimal effect on energy consumption.

The IEEE 802.15.4 standard [2] is considered as the most mature standard for WBANs with wide range of applications in health care [3]. It reasonably meets the application requirements of short range WBANs. Further, in order to deal with limited memory, energy, and processing power of sensor nodes, this standard supports appropriate physical and MAC layers. A WBAN is typically based on three types of topologies including star, peer-to-peer, and mesh [4]. Star topologies are suitable for limited range and small networks and rely on a coordinator to coordinate sensor nodes. These topologies are generally useful for networks, where energy efficiency is the prime concern. Peer-to-peer topology also requires a WBAN coordinator, where devices can communicate with each other, if they are within the communication range of each other. Cluster tree topology is similar to peer-to-peer topology; however it is relatively complex to implement. In this topology, root acts as a coordinator while remaining nodes act as the tree leaves. In this topology, sensors nodes within the transmission range of each other are able to communicate.

In WBANs, different factors for failed frame transmissions include distance, transmission range, channel conditions, interference, and medium access mechanisms. Due to critical nature of patient's data, many WBAN applications require significant reliability of transmissions. For example, a typical medical application requires above $99 \%$ delivery ratio [5]. In order to achieve that high level of reliability, an efficient 
retransmission scheme needs to be employed. However, an efficient retransmission scheme which is able to schedule retransmissions is a nontrivial issue; to be effective it must consider the number of failed transmission attempts by each node; otherwise it may further deteriorate the performance of a WBAN. A naive and poorly designed retransmission scheme may further result in collisions and delays and may result in waste of energy.

The CSMA/CA mechanism used by IEEE 802.15.4 is considered suitable to conserve energy by sensing the channel prior to transmission and therefore avoids large number of collisions. In CSMA/CA, a node always makes sure that the channel is idle before initiating the transmission. It is controlled by backoff periods, where an initial backoff period is selected from the contention window. Each node performs clear channel assessment (CCA) once its backoff period is expired. However, if two or more sensor nodes try to transmit during the same time slot, it may result in a collision, triggering a retransmission retry. If maximum number of retransmission retries exceeds a predefined retransmission limit, the packet is dropped. However, if retransmission attempts from different sensor nodes are not appropriately prioritized, it may further result in more collisions. These collisions can lead to loss of vital information, which can be fatal to a patient [6]. Although CSMA/CA is a simple subtle multiple access technique to avoid collisions and works efficiently when channel conditions are favorable, however, it provides no time guarantee for retransmission of a failed packet [7]. In the worst case, a failed frame may be discarded after maximum number of retransmission attempts and therefore incurs extra overhead. There are studies, which evaluate the efficiency of CSMA/CA in IEEE 802.15.6 in terms of energy efficiency, delay, and throughput under saturated traffic conditions [8-11]. These studies reveal that the reliability of CSMA/CA is adversely affected under high traffic loads, thus affecting average network throughput, latency, and energy consumption.

Majority of the MAC protocols designed for WBANs either do not consider retransmissions at all $[3,5,12]$ or just favor an early retransmission attempt for each of the failed packets [13]. The work proposed in [14] employs a time division multiple access- (TDMA-) based approach to use the spare slots for retransmissions. However, this scheme does not consider the number of failed transmissions by each node and rely on spare slots. Under high traffic, the number of spare slots is not sufficient to accommodate retransmissions, which may further deteriorate the performance of a WBAN. Several other recently proposed retransmissions schemes which consider improving the reliability of WBANs include Block Ack [15], Flipping [16], Master/Slave [15], LReAck [17], Split Decision [18], and ASA [19].

Block Ack scheme modifies TDMA frame to schedule retransmissions with the help of negative acknowledgment (NAck), whereas ASA utilizes spare slots to schedule them. Master/Slave technique is based on polling and rely on a coordinator to schedule retransmissions. In split decision, retransmissions are scheduled by partitioning the payload into different partitions, and appending CRC-8 in each of them. In flipping strategy, regular transmission slots and slots for retransmission are clumped to increase the probability of successful transmissions. Another approach RTDMA exploits the use of temporal diversity and achieves reliability by transmitting the same packet twice. Although these retransmission schemes target improving reliability of a WBAN, however they are not adaptive to each node's individual failed transmissions, which greatly influences the effectiveness of these frame retransmission schemes at the expense of increased retransmission cost.

To tackle the aforementioned problems with the retransmission mechanism of CSMA/CA and with other retransmission schemes, we propose a retransmission adaptive intelligent MAC (RAI-MAC) protocol for fair retransmissions in a WBAN. The proposed retransmission adaptive MAC exploits the number of failed transmission attempts by each of the sensor nodes. This information enables the coordinator to decide about the retransmission class of each sensor node in the WBAN. This retransmission class controls the next backoff interval of all current transmitting sensors in a WBAN, thus affecting the retransmission priority of each node. Due to adaptive adjustment of backoff interval, RAIMAC is able to reduce the probability of collisions, and delay as experienced by the CSMA/CA, and other retransmission schemes. The simulation results show that the proposed scheme can effectively use the number of failed transmissions for efficient MAC retransmissions. Our scheme significantly improves the average network throughput and average transmission delay with reasonable energy consumption.

The remaining part of the paper is structured as follows: Section 2 is devoted to some of the retransmissions related studies in WBAN available in literature. Section 3 briefly describes the IEEE 802.15.4 standard. Section 4 provides a detailed insight into the operation of IEEE 802.15.4 CSMA/CA mechanism. Section 5 provides the motivation to our work. Section 6 presents the adaptive retransmission mechanism, i.e., RAI-MAC protocol based on IEEE 802.15.4. Section 7 verifies the performance of RAI-MAC and compares it with the CSMA/CA mechanism used by IEEE 802.15.4 and Block Ack. Finally, concluding remarks are given in Section 8.

\section{Related Work}

Reliability of medical applications is considered highly critical compared to nonmedical applications. In order to achieve high reliability, appropriate retransmission mechanisms need to be employed. Recently, in IEEE 802.15.6 standard proposals [13] an early retransmission of failed frame is recommended; however no appropriate retransmission mechanism has been explained.

Several MAC based retransmission schemes have been proposed in literature. These schemes aim to improve the reliability of IEEE 802.15.4-based WBANs. One of the most naive approaches is TDMA-based fixed slot allocation [20]. In this approach, nodes access the channel in their allocated time slots in the next superframe and therefore experience no contention. TDMA performs well as it is subject to no collisions for channel access. In this scheme, a retransmission is more likely required due to a change in gesture or shadowing effect. 
However, no retransmission mechanism is employed in this scheme to tackle failed transmissions affecting its throughput. Authors in [21] propose a variable TDMA allocation scheme to mitigate error rate in WBAN. The same authors [16] extend the idea of variable TDMA allocation by using a flipping scheduling strategy. The flipping strategy schedules the transmissions in two rounds. The successful transmissions observed in the previous superframe are scheduled in the first round, whereas, in the second round, regular transmissions and retransmission are clumped to increase the probability of successful transmissions. The authors in [14] propose an efficient TDMA-based retransmission mechanism by using spare slots. The experiments conducted by the authors reveal that the proposed scheme improves network reliability while achieving energy efficiency in scheduled access mode.

The Block Ack scheme is based on TDMA approach where TDMA frame is divided into two parts. From the first part each node is assigned a time slot to send data to the coordinator. Coordinator broadcasts a special frame called a Block Ack frame, which carries one bit per node. This bit is set to 1 to mark acknowledgment (Ack) for successful packet reception and to 0 to denote negative acknowledgment (NAck). The second part of the TDMA frame is exclusively used by the coordinator to schedule retransmissions of nodes with NAck. Upon receiving the Block Ack, a node retries to transmit packet in its allocated time slot in the TDMA frame. A node receives a Block Ack frame message based on its position during the cycle. If no message is lost during the first part of the TDMA frame, the frame continues with one slot allocation per sensor node, plus one. However, in case of a failed transmission, the period of a second chance is equivalent to the TDMA cycle, and therefore the frame doubles the number of slots, plus one. The Block Ack scheme performs well in terms of energy efficiency and channel contention as compared to the individual acknowledgments by exploiting temporal diversity. It is the fastest way to distribute acknowledgments, which are used for fast retransmissions. However, unlike TDMA, Block Ack may increases the average jitter experienced by the network. Further, a lost Block Ack may severely impair the reliability of a WBAN [15].

In Master/Slave technique also called polling technique, coordinator explicitly sends requests to sensor nodes to transmit their data. Upon receiving this request, nodes send the data to the coordinator. If coordinator does not receive the data packet from the sensor node within the specified time, it requests the sensor node to retransmit it [15]. In Redundant TDMA (R-TDMA) nodes exploit the use of temporal diversity and transmit a data packet twice. In this scheme, a frame is divided into two TDMA blocks and each node is allocated one slot from each of the blocks. Unlike Block Ack, R-TDMA does not rely on any acknowledgments for retransmissions; if packet is received within two allocated time slots, communication is considered successful [15].

In adaptive scheduling algorithm (ASA) [19], nodes are allocated spare time slots for immediate retransmissions in order to reduce average transmission latency as compared to the Master/Slave technique. Similar to R-TDMA, this scheme does not use beacon frames for synchronization and association control. Rather, it inserts retransmission slots information in the acknowledgment of request. Coordinator checks and if the number of requested slots by a node is less than $50 \%$ of the total slots in the superframe, coordinator allocates the requested time slot and spare slot, both one to one ratio. If the number of requested slots is greater than $50 \%$ and less than $66 \%$, coordinator allocates both the requested and spare time slots, two to one ratio. If the number of requested slots is greater than $66 \%$, coordinator allocates the requested slots only.

Authors in [22] propose a windowless block acknowledgment scheme, which guarantees continuous packet retransmissions irrespective of the underlying link unreliability. This scheme is based on a bursty convergecast scheme. Sender node organizes its packet queue in form of a linked list. Most recent packets with smaller retransmissions count receive higher transmission priority. If a node does not have any new packets to transmit, it immediately retransmits the unacknowledged packets. Similar to Block Ack, successfully received group of packets are acknowledged by using a block acknowledgment.

Authors in [23] introduce a polling-based approach similar to Master/Slave scheme. However, compared to the Master/Slave which has no limit on the number of retransmissions, this scheme limits number of retransmissions. Further, compared to the Master/Slave approach, this approach is based on earliest deadline first (EDF) scheduling to schedule real-time retransmissions. Other polling-based techniques include queued retransmissions (QR) and adaptive $Q R$ (AQR) [24]. In QR, a coordinator schedules transmissions in two rounds. In the first round, coordinator transmits data, whereas in the second round it schedules any pending retransmissions. In QR scheme, a node with successful transmission favors other nodes with higher number of pending retransmissions. Compared to $\mathrm{QR}, \mathrm{AQR}$ uses a longer history of successful transmissions of a node.

In [25], a retransmission scheme based on network coding and time diversity has been proposed. In this scheme, if a packet is not received by a node within the specified time, it immediately transmits a negative acknowledgment to source node. Sender uses an encoded maximal set of lost packets to retransmit to multiple destinations simultaneously. Receiving nodes decode their message from the received encoded set in order to retrieve the lost packet.

Further to CSMA/CA contention problem, recently two approaches called CSMA/CA algorithm adaptation [26-28] and CSMA/CA algorithm with retransmission adaptation [5] have been proposed in literature. In CSMA/CA with adaptation [26], a collision-aware backoff algorithm has been introduced to update the value of backoff dynamically according to collision rate in order to reduce energy consumption and transmission latency. A memorized backoff scheme (MBS) based on exponential weighted moving average has been proposed in [28]. The scheme adjusts the contention window dynamically based on the current traffic load and therefore shows significant throughput improvement. The authors in [27] improve successful transmissions probability by sensing the channel. In their scheme, nodes sense channel according to the order assigned by the coordinator, where channel is partitioned into time slots. Further, authors use 
a modified backoff algorithm to reduce the number of collisions. However, their backoff algorithm does not take into account dynamic network conditions. Further, this scheme does not provide any guaranteed retransmission mechanism to reduce the number of collisions. In retransmission adaptive approach to CSMA/CA, authors consider the retransmission of critical messages. In a similar technique in [5], authors consider the use of retry transmit (RT) field in the MAC control field. A failed transmission sets this field to one. In the next superframe, nodes with the RT field set to one are allocated GTS by the coordinator to guarantee retransmissions.

In another retransmission approach called split decision [18], authors propose the idea to split the payload into 3 partitions and insert a CRC-8 error check code at the end of each partition. In the first cycle, sender transmits partitioned data. If an error is detected in any of the partitions, receiver replies back with a NAck along with the partition information. Second cycle is dedicated to the recovery of partitions by using CRC-16 for which sender has received a NAck. If receiver detects no error in the recovery partitions, it performs partial recovery without CRC-8 after Ack transmission.

Although all the schemes reviewed above try to achieve reliability by retransmitting failed packets, however these schemes do not use any adaptive coordinator-controlled strategy to schedule the retransmissions. Majority of the schemes either schedule their retransmissions at their earliest, like EDF [23], or use spare slots to schedule any pending retransmissions, e.g., ASA [19]. These schemes do not consider any retransmission classification and prioritization to schedule the retransmissions and may result in collisions, thereby degrading the reliability of the network. Further, a nonprioritized backoff algorithm used by the IEEE 802.15.4 may incur longer delay and higher energy consumption. There are some retransmission schemes like QR, which consider the number of pending retransmissions to retransmit; however these schemes lack any appropriate coordinatorbased classification and prioritization, affecting their performance. Hence, in this paper, we propose an adaptive retransmission scheme based on IEEE 802.15.4 to prioritize the retransmissions dynamically by the WBAN coordinator. Depending on the traffic conditions, this coordinator-assisted priority-based retransmission strategy allows the nodes to fine-tune to the most appropriate backoff period in order to prioritize their next retransmission attempt.

\section{An Overview of IEEE 802.15.4}

IEEE 802.15.4 can operate in beacon-enabled and beaconless modes. In the beaconless mode, no beacon is used between the coordinator and sensor nodes for synchronization. In this mode, access to channel is based on unslotted CSMA/CA mechanism. Mostly, this mode is used by the infrastructureless mesh topologies, where no synchronization is required for communication between the coordinator and sensor nodes [29]. On the other hand, in the beacon-enabled mode, coordinator transmits a superframe consisting of active and inactive periods, which are bounded by the beacons. The active part of the superframe is further subdivided into CAP and contention-free period (CFP) [30, 31]. During the inactive period, nodes tend to sleep to conserve energy. Access to the CAP period is controlled through slotted CSMA/CA mechanism [32], whereas access to the CFP period is guaranteed by the coordinator through guaranteed time slots (GTS) allocation. Both the star and cluster tree topologies operate in the beacon-enabled mode, where tight synchronization is required for communication between the coordinator and sensor nodes. The non-beacon-enabled mode of IEEE 802.15.4 uses no superframe structure. All devices in this mode are always active and ready to transmit by using unslotted CSMA/CA. Therefore, this mode experiences higher number of collisions as compared to the beaconenabled mode.

The superframe structure is illustrated in Figure 1 [33]. As it can be observed from Figure 1, an IEEE 802.15.4 superframe is bounded by special synchronization packets, called beacons. A beacon interval (BI) denotes the interval between two consecutive beacons and is calculated as follows: $B I=$ aBaseSuperframeDuration $\times 2^{B O}$ symbols, where $B O$ denotes the beacon order. Further, it is clear from Figure 1 that each superframe consists of active period and inactive period. The active period of the superframe also referred to as superframe duration (SD) is controlled by the superframe order ( $\mathrm{SO}$ ) and is calculated as $S D=$ aBaseSuperframeDuration $\times 2^{S O}$ symbols. WBAN sensor nodes tune to low power modes during the inactive period to conserve energy. The SD is further divided into two periods consisting of 16 slots: CAP and CFP. During the CAP, nodes contend for medium access by using slotted CSMA/CA, whereas CFP ensures contention-free channel access through GTS based on TDMA.

\section{CSMA/CA Operation in IEEE 802.15.4-Based WBANs}

IEEE 802.15.4-based WBANs use CSMA/CA to access medium during the CAP period. CSMA/CA supports WBANs based on different topologies including cluster tree topology, star topology, and peer-to-peer topology. It can be used both in the beacon-enabled and in the non-beacon-enabled modes. The first mode uses the slotted CSMA/CA while the second mode is based on the unslotted version of CSMA/CA. In the beacon-enabled mode, in order to contend the channel during the CAP, a node maintains three variables: binary exponent $(B E s)$, contention window $(\mathrm{CW})$, and a number of backoff stages (NB). A node selects its backoff period called $\mathrm{BP}$ randomly from $\left(0,2^{B E-1}\right)$ before initiating a transmission. The value of $B E$ is selected as follows: $B E \in($ macMinBE, macMaxBE) with an initial value of macMinBE. Once BP expires, a node performs two CCA checks to sense the channel. If channel is idle, node transmits the packet and waits for an acknowledgment from the coordinator. Conversely, if channel is busy, node increments the values of $\mathrm{NB}$ and $B E$. In the IEEE 802.15.4 standard, the values of macMinBE and macMaxBE are set to 3 and 5, respectively. If the values of $B E$ and $\mathrm{NB}$ exceed their maximum limit, i.e., aMaxBEs and macMaxCSMABackoffs, respectively, the packet is dropped 


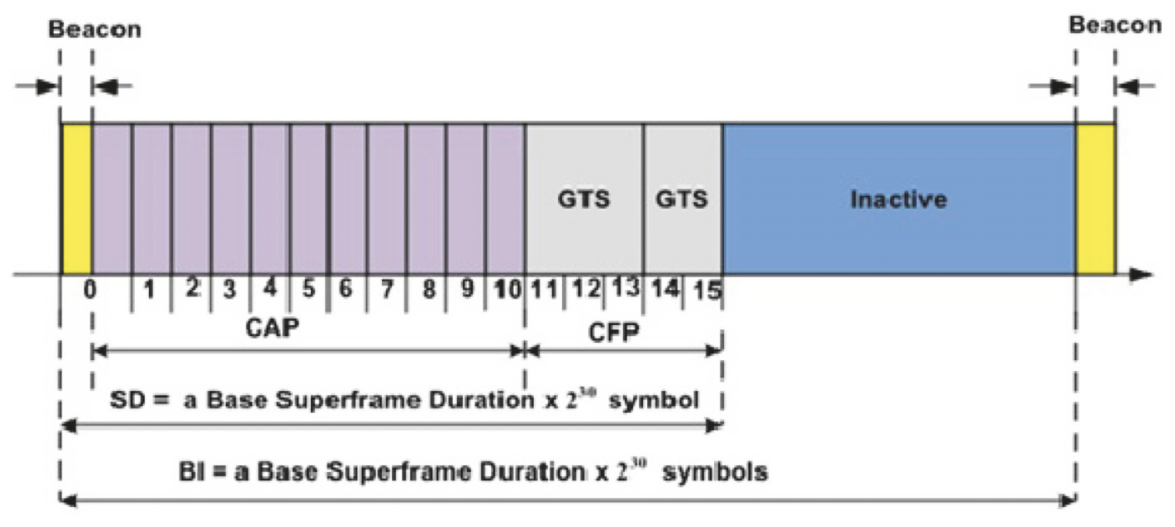

FIGURE 1: IEEE 802.15.4 frame structure [33].

without further transmission attempts. In the standard the value of macMaxCSMABackoffs is defined as 4 [35].

\section{Motivation}

Although CSMA/CA is the most popular and widely used medium access mechanism with low complexity, however, it may experience serious consequences under emergency situations where nodes may fail to transmit critical information to the coordinator, thereby impairing the network efficiency. The CSMA/CA used by IEEE 802.15.4 is based on a very limited range of $B E$ values, i.e., $B E=$ macMinBE$\operatorname{aMaxBE}$, where macMinBE $=3$ and the $\operatorname{aMaxBE}=5$. Due to this limited number of $B E$ values, nodes in a WBAN may select the similar BP resulting in higher number of collisions. Under emergency situations, a collided packet may contain critical information about a patient's condition, e.g., stroke or heart rate information, which can be very fatal for the patient.

The main factors which can affect the performance of CSMA/CA algorithm are values of $B E$, i.e., BEmin and BEmax, the number of CCA attempts prior to transmission, the strategy to adjust $B E$ for each subsequent collision or successful transmission, and the maximum number of retransmission retries. The existing CSMA/CA mechanism uses a backoff mechanism which strictly depends on BEmin and BEmax and is controlled by each node individually with no control from the coordinator. WBAN nodes may experience different retransmission delays depending on the traffic conditions. Under high traffic loads, a node experiencing more collisions has to back off longer to retransmit the lost packet as compared to the nodes with better medium access. Further, WBAN nodes based on CSMA/CA use no prioritized backoff algorithm to attempt for retransmissions which may severely impair the average network throughput, energy efficiency, and delay.

In this work, we plan to fine-tune the backoff value of each node according to its priority class during the CAP access. Node priority classes in our proposed scheme are defined and controlled by the coordinator. Depending on the number of failed transmissions, coordinator dynamically decides about the priority class of each node, whenever either number of nodes or traffic load in the network changes.
Therefore, unlike existing CSMA/CA mechanism and other retransmission strategies, coordinator has full control over the retransmission priority of each node. Each sensor node tunes its BEmin value for any early medium access for retransmissions as dictated by its retransmission priority class.

\section{Retransmission Adaptive Intelligent MAC (RAI-MAC)}

In RAI-MAC, coordinator decides the retransmission class of a node depending on the number of unsuccessful transmission attempts. Once retransmission class is decided by the coordinator, a RAI-MAC-enabled node dynamically adjusts its backoff time according to the class. The design of RAI-MAC is explained under retransmission classification, retransmission map, and beacon modification and is illustrated below.

\subsection{RAI-MAC Design}

Traffic Estimation and Retransmission Classification. In RAIMAC, the coordinator estimates the number of failed transmissions of each sensor node observed during the last superframe. A sensor node adds an extra bit in the header of a data frame denoted as Retrans ${ }_{b}$ to indicate data as retransmission; i.e., a Retrans ${ }_{b}$ bit set to 1 indicates a data frame transmission as retransmission, whereas a bit 0 shows it as a transmission. Coordinator calculates the number of failed retransmissions by counting the data frames with Retrans R $_{b}=1$ for each node.

After traffic estimation, it assigns different retransmission

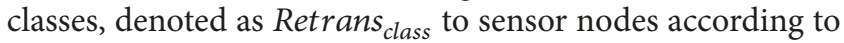
the number of failed transmissions, denoted as Trans failed $_{\text {, }}$ which are observed in the last superframe. The criteria used by the coordinator for retransmission classification for the next superframe is based on Table 1. Table 1 classifies a node according to the number of Trans failed. If Trans $_{\text {failed }}$ of a node exceeds a predefined maximum threshold, i.e., MaxThreshold, the node is assigned Retrans class $1_{1}$ if Trans failed $_{\text {Th }}$ is more than a predefined threshold, called MidThreshold, and less than MaxThreshold, node is placed in Retrans class $2_{2}$; 
TABLE 1: RAI-MAC retransmission classes.

\begin{tabular}{lr}
\hline Number of Failed Transmissions & Retransmission Class \\
\hline Trans $_{\text {failed }}>$ MaxThreshold & Retrans $_{\text {class } 1}$ \\
\hline Trans $_{\text {failed }}>$ MidThreshold\& Trans & failed \\
\hline Trans $_{\text {failed }}>$ MinThreshold\& Trans & failed \\
\hline
\end{tabular}

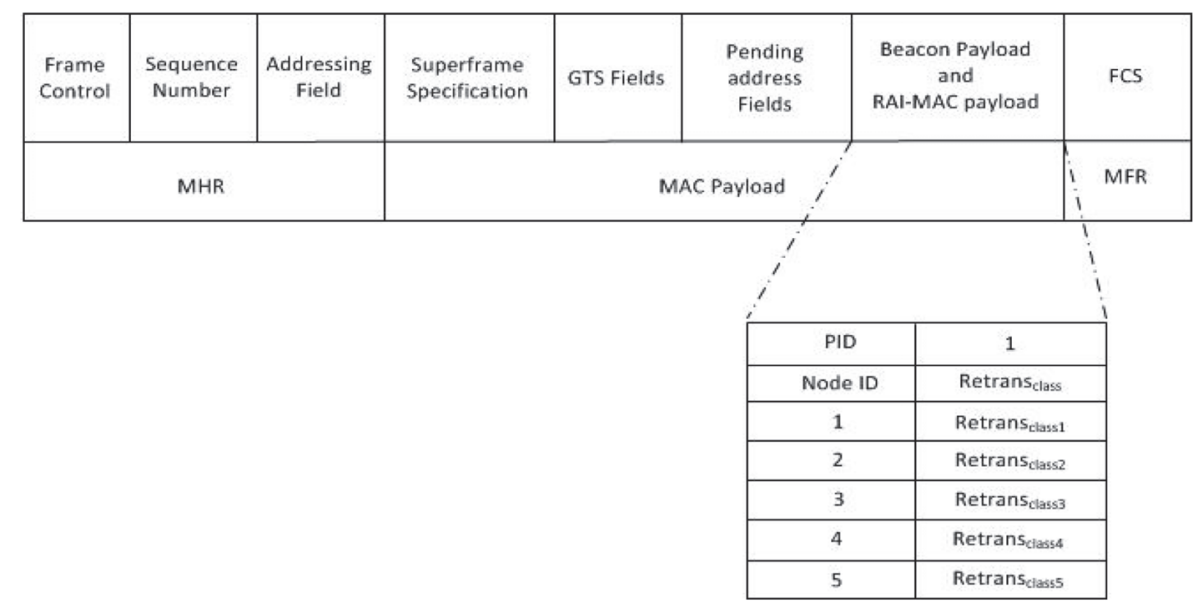

FIgURE 2: RAI-MAC beacon [34].

TABLE 2: R-MAP structure with sample entries.

\begin{tabular}{lc}
\hline PID & 1 \\
\hline Node ID & Priority Class \\
1 & Retrans $_{\text {class } 1}$ \\
\hline 2 & Retrans $_{\text {class } 2}$ \\
\hline 3 & Retrans $_{\text {class } 3}$ \\
\hline 4 & Retrans $_{\text {class } 2}$ \\
\hline 5 & Retrans $_{\text {class } 1}$ \\
\hline
\end{tabular}

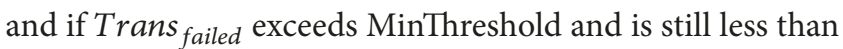
MidThreshold, it is classified as Retrans class $3_{\text {. }}$.

Retransmission Map (R-MAP). In RAI-MAC, the coordinator maintains a list for retransmission classifications, we call it retransmission map (R-MAP). R-MAP structure is illustrated in Table 2. R-MAP carries information about the priority identification field (PID), node id, and priority class. The PID is set to either 1 or 0 ; field 1 indicates that R-MAP carries information about the priority class; field 0 indicates that R-MAP contains no information about any of the priority classes. Further, R-MAP also contains information about the node id along with its priority class, which coordinator has decided during the last superframe according to Table 1.

RAI-MAC Beacon. Once the coordinator has computed the R-MAP list similar to Table 1, it appends it with the beacon payload and therefore incurs no extra overhead to transmit R-MAP. The modified beacon used by RAI-MAC is shown in Figure 2 [34]. It consists of three fields: MAC header
(MHR), MAC payload, and MAC footer (MFR). MHR is further subdivided into frame control, sequence number, and an addressing field. MAC payload consists of superframe specification, GTS fields, pending address fields, and RMAP payload. MFR of the beacon contains only one field, i.e., frame check sequence (FCS). The frame control field specifies the type of MAC frame, which is being transferred by the coordinator. The frame sequence number of the MHR is used to reassemble frames, which are received out of sequence, and it is also used to match the acknowledgment with the previous transmission. Addressing field in MHR consists of source and destination addresses, and it may vary from 0 to 20 bytes. The superframe specification field of the MAC payload specifies the superframe information including superframe duration, CAP, CFP, and coordinator identification, whereas its GTS field contains information about the GTS slot allocation. Pending address field in MAC payload is used by the coordinator to transfer pending data. The R-MAP payload in the RAI-MAC beacon carries RMAP list, appended by the coordinator which is used by the sensor nodes to adjust their retransmission priorities. The only field in FHR, i.e., FCS, consists of 16-bit CRC, which helps to verify the integrity of the received MAC frame by the receiver.

6.2. RAI-MAC Operation. In RAI-MAC, retransmissions are adaptively controlled by the coordinator and nodes. The coordinator decides the retransmission class of a sensor node depending on the number of failed transmissions observed during the last superframe as illustrated in Algorithm 1. A node dynamically adjusts the backoff behavior of CSMA according to the retransmission class decided by the coordinator and it is illustrated in Algorithms 2 and 3. 


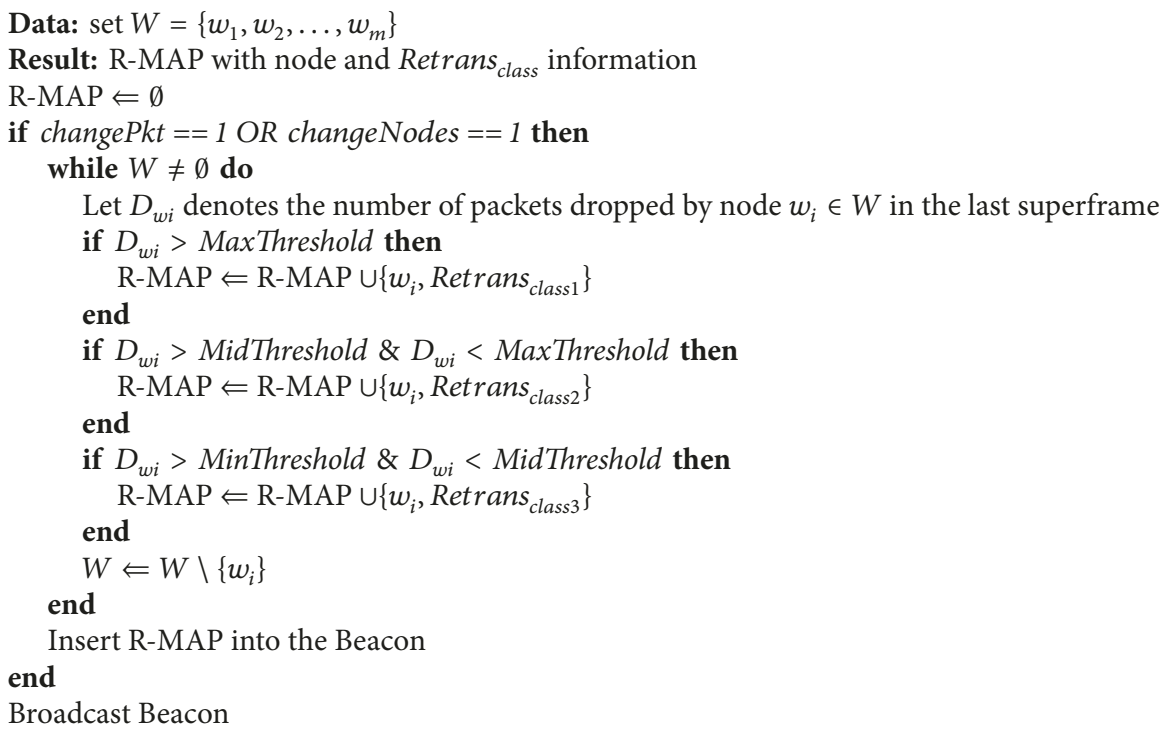

Algorithm 1: RAI-MAC at coordinator.

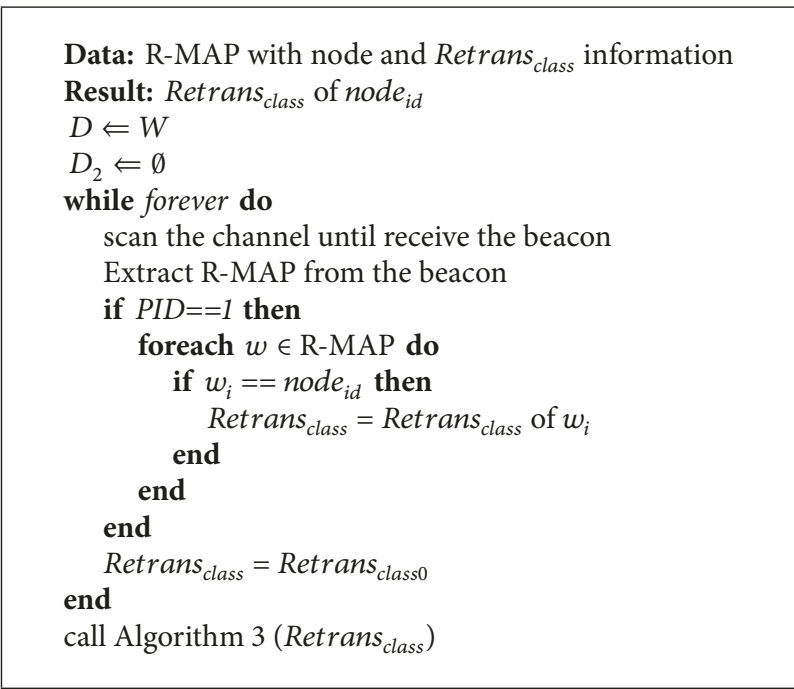

Algorithm 2: RAI-MAC at node.

RAI-MAC Coordinator Algorithm. Algorithm 1 illustrates the RAI-MAC behavior on a coordinator. Whenever coordinator detects the traffic load changes due to change in number of either nodes or packets, it calculates the number of failed transmissions, denoted as $D_{w}$, experienced by each node. The coordinator computes the R-MAP by using $D_{w}$ information and according to retransmission classes criteria defined in Table 1. Each entry of R-MAP consists of node $e_{i d}$ along with its

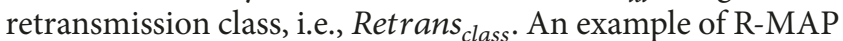
structure computed by Algorithm 1 is shown in Table 2. After computing the R-MAP, coordinator broadcasts it by inserting it into the beacon.

RAI-MAC Node Algorithm. Algorithms 2 and 3 illustrate the behavior of RAI-MAC on each sensor node. All sensor nodes in a WBAN synchronize with the coordinator by using the information received through the beacon. Upon receiving the beacon, the node extracts the PID field. The PID field confirms if the R-MAP carries any information about the retransmission classes of the nodes. If this field is set to 0 , it means R-MAP carries no information about the retransmission classes; however, if it is 1 , it indicates that R-MAP has information about the retransmission classes. Further, if value of PID is set to 0 , it helps the node to skip processing the R-MAP any further. If a node retrieves the PID field as 1, it processes the R-MAP and extracts its retransmission class if specified by the coordinator in the R-MAP. If node finds no retransmission class in the R-MAP, it specifies its retransmission class as Retrans class $0_{\text {. }}$. The retransmission class Retrans $_{\text {class } 0}$ specifies the same 


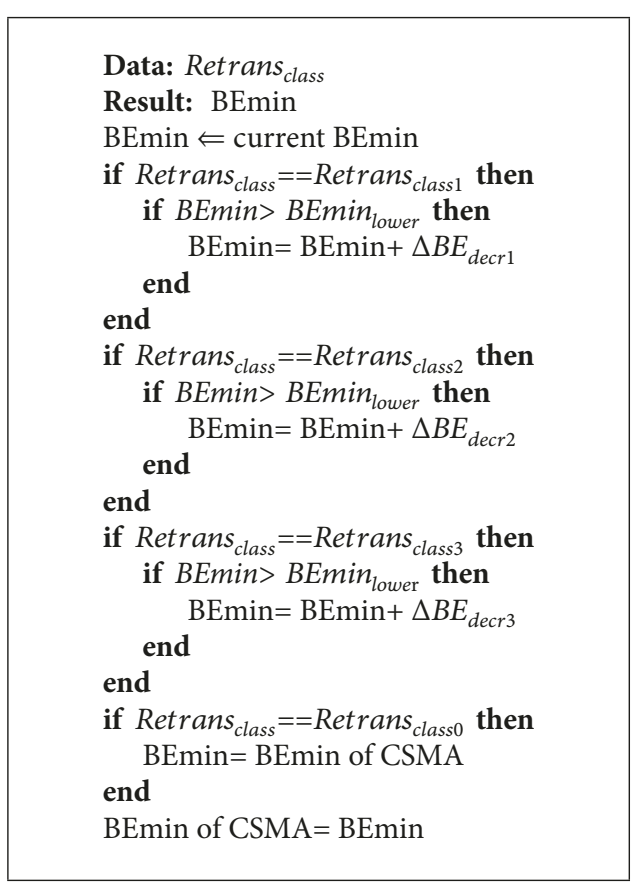

Algorithm 3: RAI-MAC at node.

backoff behavior as dictated by the default CSMA/CA mechanism.

Once a node has information about its retransmission class either extracted from the R-MP or specified as Retrans $_{\text {class } 0}$, it invokes Algorithm 3. Algorithm 3 prioritizes the channel access of each sensor node by adjusting the value of $\mathrm{BE}$ according to the priority class of a node as decided by Algorithm 2. In the IEEE 802.15.4 standard, priority to the channel access is controlled by adjusting BEmin and BEmax. However, at the earlier stage, priority access to the channel depends only on the value of BEmin, whereas BEmax controls the channel access at the final stage. Contemplating to this, Algorithm 3 adjusts only the value of BEmin to control the access priority at the earlier stage of retransmission only.

In order to prioritize Retrans class $1_{1}$, Algorithm 3 increases the value of BEmin by $\triangle B E_{\text {decr } 1}$. The Retrans class $2_{2}$ increments the value of BEmin by $\triangle B E_{d e c r 2}$ to prioritize over Retrans $_{\text {class } 3}$. The Retrans class 3 increases BEmin by $\triangle B E_{\text {decr } 3}$

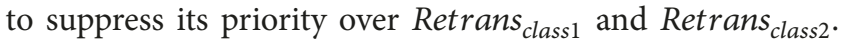
Algorithm 3 decrements the value of BEmin for all the three classes, i.e., Retrans class $1_{\text {, Retrans }}$ Rlass $2_{2}$, and Retrans Rlass $1_{1}$ only if the value of BEmin is greater than the BEmin lower, as specified by RAI-MAC, where BEmin ${ }_{\text {lower }}$ denotes the maximum possible value for BEmin.

\section{Performance Evaluation}

It is possible to evaluate RAI-MAC by using IEEE 802.15.4 compliant MicaZ nodes equipped with OpenZB microkernel [36]. However, in this paper we evaluate the performance of RAI-MAC by using ns-2.34 and compare it with the retransmission mechanism used by IEEE 802.15.4, i.e., slotted CSMA/CA and a most recent retransmission scheme, Block
Ack [15]. We have performed two major sets of experiments; the first one evaluates and compares RAI-MAC with IEEE 802.15.4 with different BEmin and BEmax values; and the second one evaluates and compares RAI-MAC with the Block Ack. The details of these experiments are illustrated in the sections below.

In all simulation scenarios, the wireless body area network used for performance evaluations is based on star topology in the beacon-enabled mode. The WBAN consists of a coordinator connected with the sensor nodes within a range of $10 \mathrm{~m}$. The coordinator broadcasts beacons at regular intervals to synchronize with the sensor nodes. In our network, sensor nodes are active during the active period of superframe and they turn their radio off to conserve energy during the inactive period. All the sensor nodes generate constant bit rate (CBR) traffic where each data packet has size of 70 bytes.

If not stated otherwise, for all the experiments we have used ATMEL energy model [37] to simulate realistic results for energy consumption. We assume that the coordinator has information about the contents and reporting time of each packet. Further, we assume that the coordinator is able to detect a change in the number of nodes and a change in data rate of each sensor node. For all the experiments, the maximum number of retransmission retries is set as 5 . Each simulation run lasts for 500 seconds. Table 3 summarizes the parameters of the simulation, if not stated otherwise.

7.1. RAI-MAC and IEEE 802.15.4. In the first set of experiments, we evaluate and compare the performance of RAIMAC with the IEEE 802.15.4. We simulate three different scenarios with continuous data reporting, varying number of nodes, and variable data rate. We evaluate the performance of both RAI-MAC and IEEE 802.15.4 in terms of average end-to-end delay, average throughput, and average energy consumption.

Scenario 1. In this scenario, we investigate the performance of RAI-MAC and CSMA/CA with different BEmin and BEmax values. The other simulation parameters used for this scenario are listed in Table 3. The settings of the energy model used for this scenario are the same as ATMEL Mote and are listed in Table 4. In this scenario, we consider a WBAN consisting of 15 nodes and a coordinator. The simulated network has 8 active CBR traffic flows, each with traffic load of 3 packets/s. Each data packet has payload of 70 bytes. We compare the average goodput, average end-to-end delay, and energy efficiency with a 95\% confident level under saturation conditions, where each node always has a packet to transmit.

Figure 3 compares the average throughput of RAI-MAC and CSMA/CA with different BEmin and BEmax values. Figure 3 confirms that the average goodput observed by RAIMAC is better than CSMA/CA observed at different time intervals. This improvement is due to adaptive adjustment to BEmin as compared to CSMA/CA with different BEmin and BEmax values. This dynamic adjustment decreases the probability of two nodes to select the same backoff value resulting in fewer collisions as compared to CSMA/CA. 
TABLE 3: Simulation parameters.

\begin{tabular}{lc}
\hline Parameter & Value \\
\hline Simulation Time & $500 \mathrm{~s}$ \\
Transmission Range & $15 \mathrm{~m}$ \\
Frequency Band & $2.4 \mathrm{GHz}$ \\
Traffic Type & $\mathrm{CBR}$ \\
Packet Size & 70 bytes \\
Sensor Nodes & 15 \\
Data Rate & $20 \mathrm{kbps}$ \\
Energy Model & ATMEL Mote \\
MaxThreshold & 75 \\
MidThreshold & 50 \\
MinThreshold & 25 \\
Source Nodes & 5 \\
BEmin & 1 \\
BO=S0 & 3 \\
$\Delta B E_{\text {decr } 1}$ & 1 \\
$\Delta B E_{\text {decr } 2}$ & $B E_{\text {decr } 3}$ \\
$B E m i n_{\text {lower }}$ & 0.5 \\
\hline
\end{tabular}

TABLE 4: Energy model.

\begin{tabular}{lc}
\hline State & Energy \\
\hline Initial Energy & 4 Joules \\
Sleep & $0.02 \mathrm{~mA}$ \\
Transmit & $14 \mathrm{~mA}$ \\
Receive & $12.3 \mathrm{~mA}$ \\
Idle & $0.4 \mathrm{~mA}$ \\
\hline
\end{tabular}

Figure 4 shows the average end-to-end delay of RAIMAC and CSMA/CA with different BEmin and BEmax values. As can be observed, for smaller simulation time RAIMAC has higher end-to-end delay as compared to CSMA/CA due to its dynamic adaptation procedure. However, as the simulation time increases, more contention is in the network. In this case, the end-to-end delay experienced by RAI-MAC is competitive to CSMA/CA for its $\mathrm{BEmin}=2$ and $\mathrm{BEmac}=7$, which is mainly due to optimal backoff experienced by the sensor nodes.

Figure 5 presents energy consumption of both RAI-MAC and CSMA/CA. It is clear from the figure that RAI-MAC is competitive to CSMA/CA in terms of energy consumption for $\mathrm{BEmin}=2$ and $\mathrm{BEmax}=7$. As compared to the static BEmin and BEmax values as used by CSMA/CA, RAI-MAC adjusts BEmin dynamically according to the retransmission class as specified by the coordinator. This dynamic adaptation has lower number of collisions than CSMA/CA, thereby incurring less energy.

Scenario 2. In second scenario, we evaluate the performance of RAI-MAC and CSMA/CA by simulating 15 sensor nodes with 8 active CBR flows. We set the simulation parameters according to the table as shown in Table 3 . The power

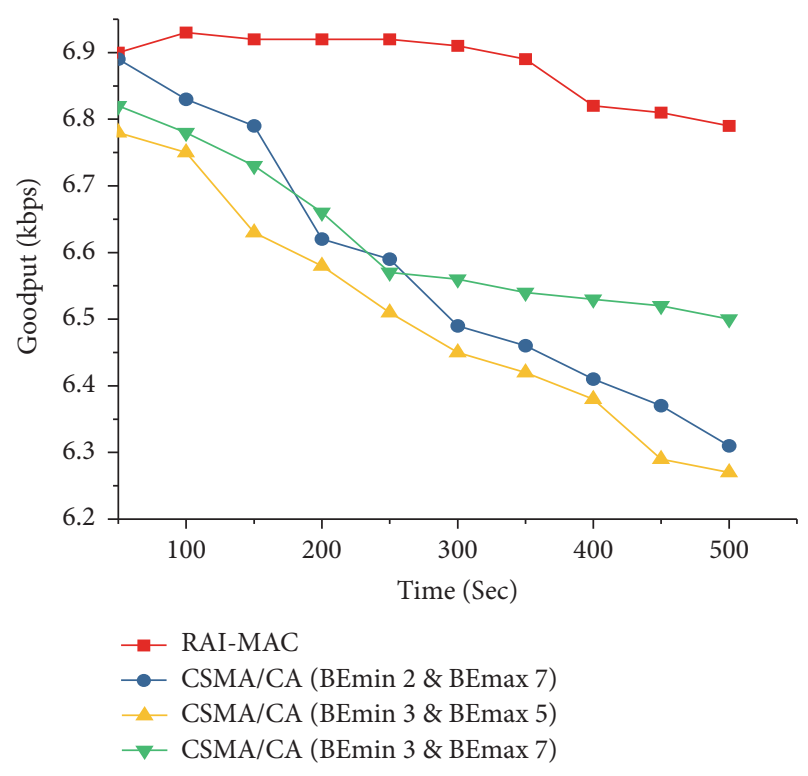

FIGURE 3: Scenario 1: average network goodput.

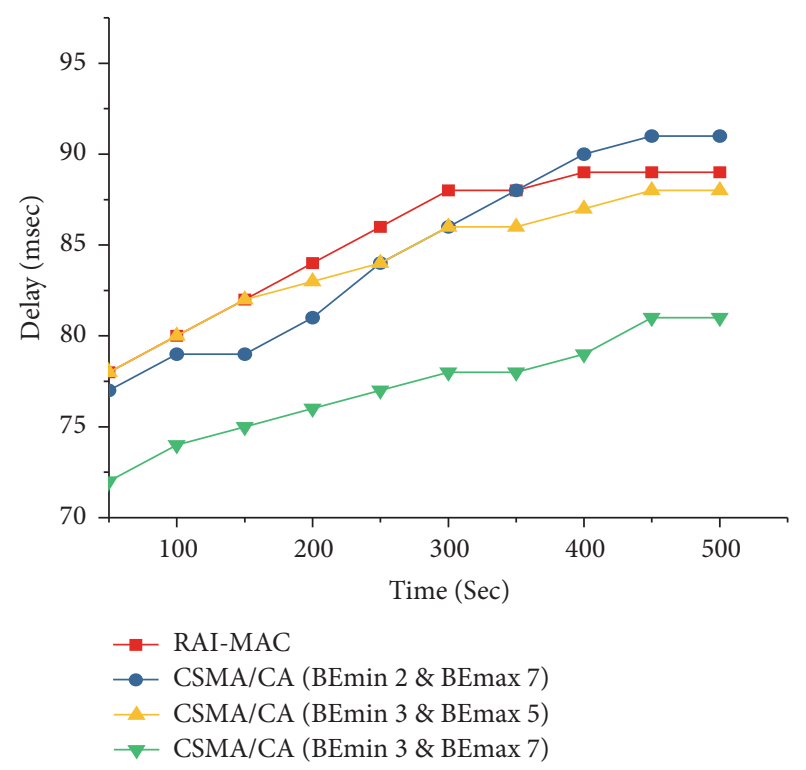

FIgURE 4: Scenario 1: average end-to-end delay.

consumption behavior of each sensor node is presented in Table 4.

Figure 6 presents average goodput for both the RAI-MAC and CSMA/CA, when traffic load varies from 0 to 3 packets/s. Other simulation parameters are the same as Table 3. It is clear from the figure that traffic load has significant effect on the achieved average network goodput. For higher traffic loads, RAI-MAC shows a clear improvement in average network goodput over CSMA/CA for all of its BEmin and BEmax values. This behavior is mainly attributed to the RAI-MAC 's dynamic adaptation to failed transmissions, thus avoiding collisions, therefore improving the goodput. An end-to-end delay sensitivity plot of RAI-MAC and CSMA/CA for varying 


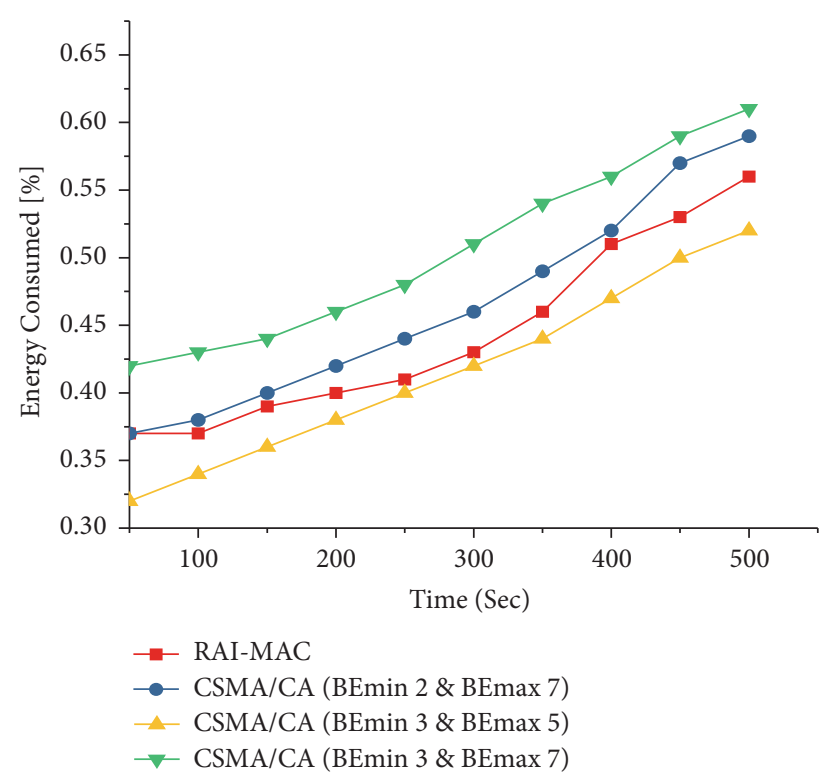

Figure 5: Scenario 1: average energy consumption.

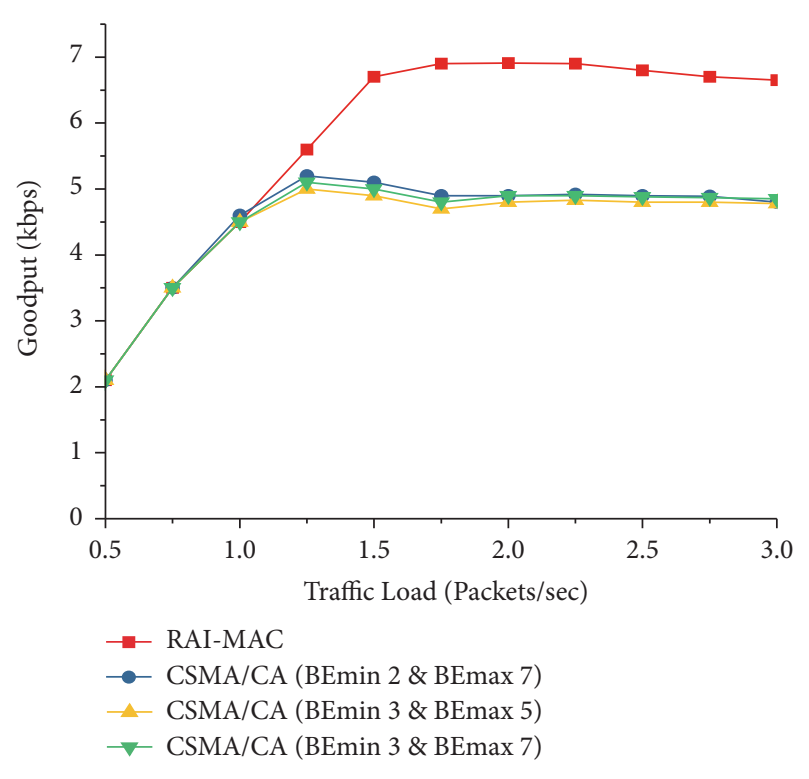

FIGURE 6: Scenario 2: average network goodput.

traffic load is shown in Figure 7. Figure shows that the traffic load has a direct influence on average end-to-end delay. The average end-to-end delay tends to increase for both the RAIMAC and CSMA/CA (for all of its BEmin and BEmax values) as the traffic load increases. However, as can be observed, RAI-MAC has significantly less average end-to-end delay as compared to CSMA/CA for all of its BEmin and BEmax values, when the traffic load exceeds 1 packet/s. This is due to the fact that RAI-MAC suffers in fewer collisions due to its adaptive backoff mechanism controlled by the coordinator.

Figure 8 illustrates the energy consumption for varying traffic loads. As can be seen, the average energy consumption experienced by a node increases with an increase in traffic

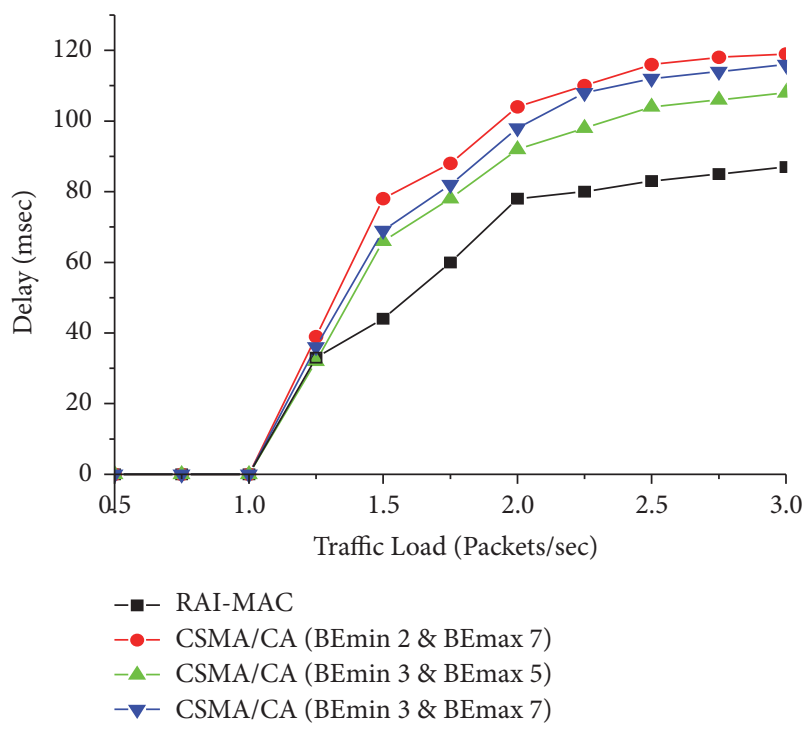

FIGURE 7: Scenario 2: average end-to-end delay.

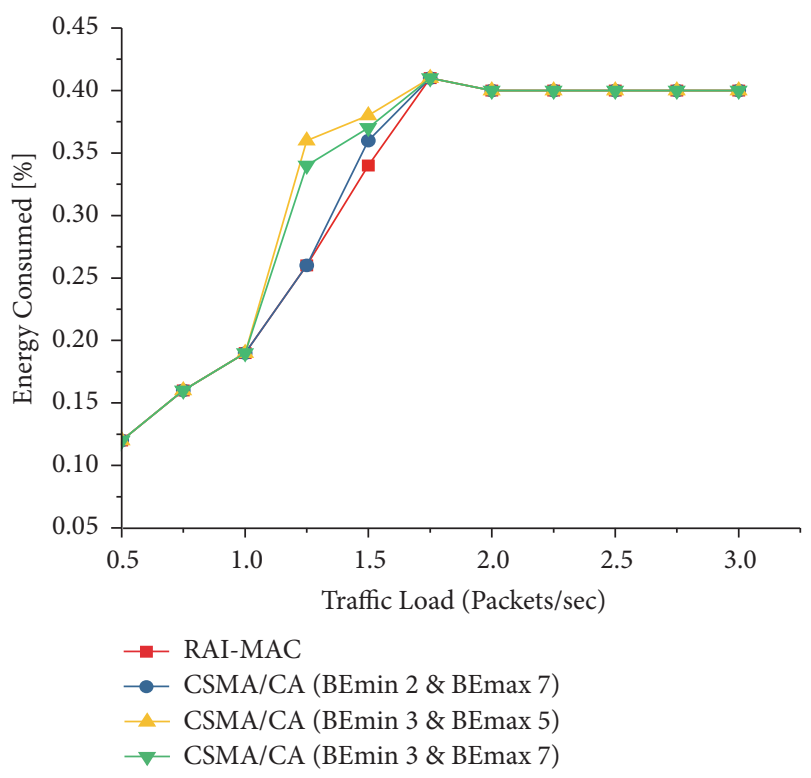

FIGURE 8: Scenario 2: average energy consumption.

load. The higher energy consumption of both the RAI-MAC and CSMA/CA is due to an increase in contention level. It can be observed that RAI-MAC has almost similar energy consumption performance as of CSMA/CA under varying traffic loads.

Scenario 3. In this scenario, we analyze the performance of IEEE 802.15.4 and RAI-MAC under different number of nodes. We vary the number of nodes from 5 to 30, where each node generates 3 packets/s. The scenario is simulated by using the star topology in the beacon-enabled mode. Other simulation parameters are the same as listed in Table 3. Each node dissipates the energy according to Table 4. 


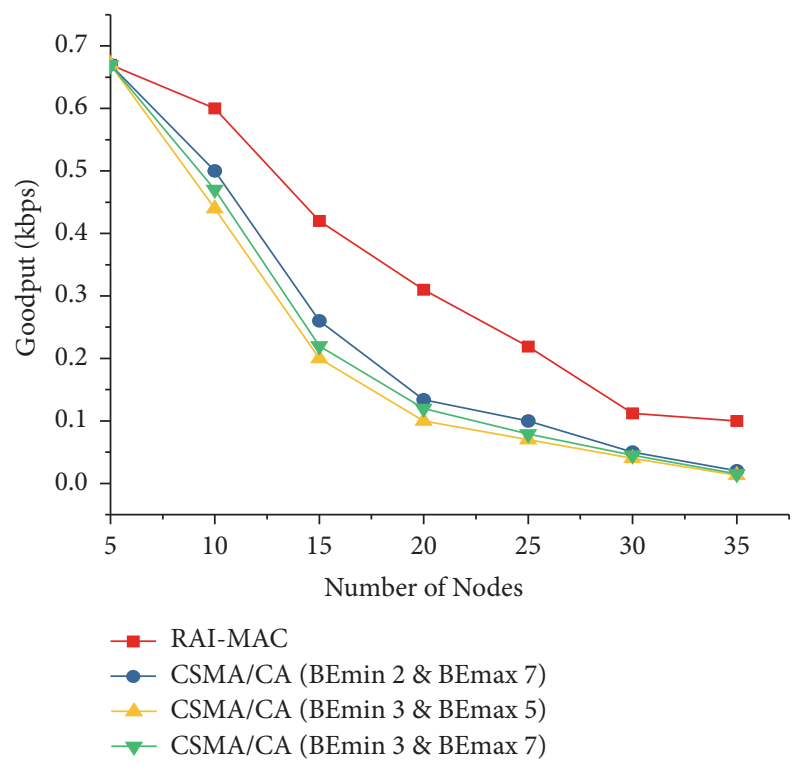

FIGURE 9: Scenario 3: average network goodput.

Figure 9 shows the average goodput of RAI-MAC and CSMA/CA versus the number of nodes. When number of nodes is less than 10, the average goodput performance of RAI-MAC and CSMA/CA is the same. However, as the number of nodes increases, the average goodput performance gap tends to increase due to high traffic volume. In RAIMAC, nodes with higher number of failed transmissions are able to access the channel frequently due to dynamic backoff adjustment, resulting in better average goodput performance. All the nodes using CSMA/CA backoff mechanism try to access the channel by using the same BEmin. Hence, number of collisions tends to increase degrading the average goodput. Figure 10 shows the average end-to-end delay experienced by both the RAI-MAC and CSMA/CA, as a function of number of nodes. From figure, it is obvious that the proposed RAIMAC shows a reasonable delay performance improvement over the CSMA/CA and for all of its BEmax and BEmin values. This is due to the fact that RAI-MAC adjusts the BEmin value according to the level of collisions, while larger values of BEmin and BEmax may cause higher average backoff delay.

Figure 11 depicts the energy consumption performance of both the RAI-MAC and CSMA/CA as a function of varying number of nodes. It is clear from the figure that RAI-MAC has competitive energy consumption as of CSMA/CA for all of its BEmin and BEmax values. The adaptive backoff procedure of RAI-MAC reduces the probability of the same backoff interval by different nodes, and therefore most of the packets are successfully delivered. As a consequence, a lower number of retransmissions are required, which improves average energy consumption.

7.2. RAI-MAC and Block Ack. In this section, we present performance evaluation of RAI-MAC and compare it with Block Ack and IEEE 802.15.4 in terms of average end-toend delay, average goodput, and average energy consumption.

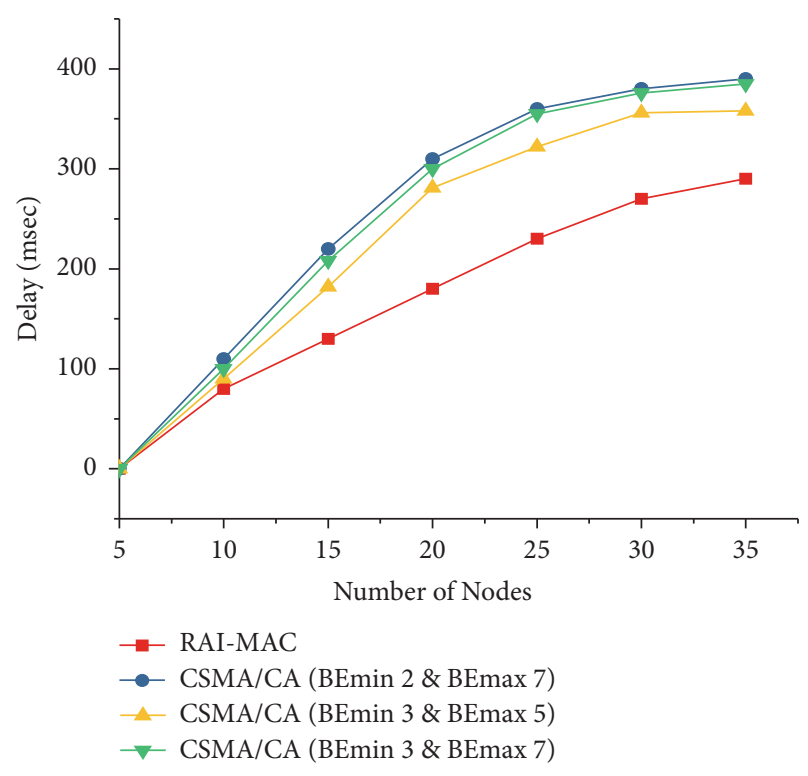

Figure 10: Scenario 3: average end-to-end delay.

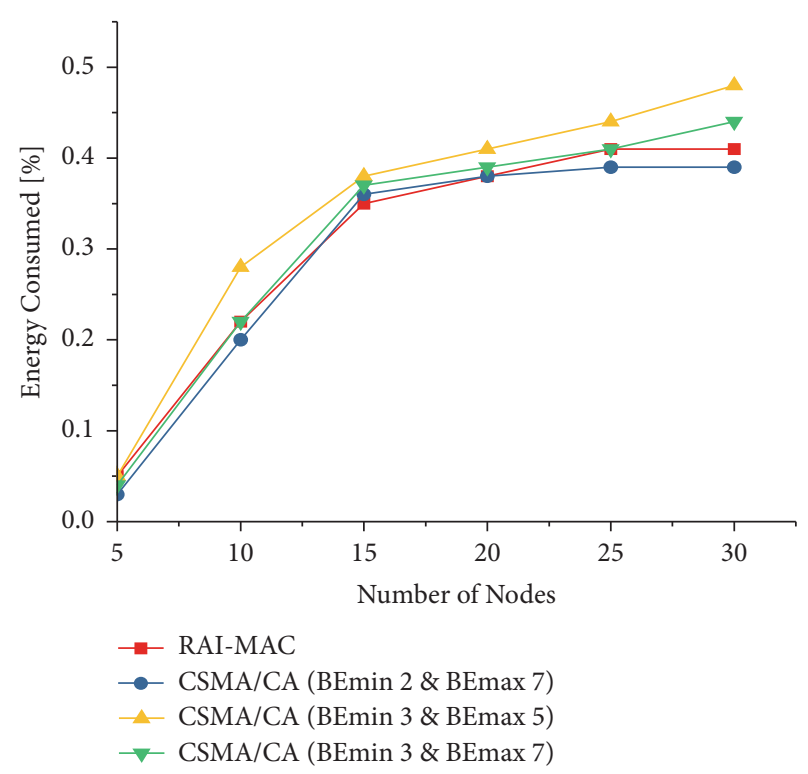

FIGURE 11: Scenario 3: average energy consumption.

In this scenario, we simulate a network of 15 sensor nodes, where each node can generate packets from 3 packets/s. Other relevant simulation parameters along with their values are listed in Table 3. Energy consumption of each node is derived from the ATMEL Mote and is the same as listed in Table 4.

Figure 12 portrays the average goodput as a function of simulation time. It can be observed from this figure that average goodput of RAI-MAC is better than Block Ack for all of the simulation time values. This difference becomes more significant for the longer simulation time as the network gets more saturated, thereby affecting the goodput of all of the medium access techniques. This means that, under saturation conditions, packets transmitted by RAI-MAC are mostly likely able to reach their destination due to lower number 


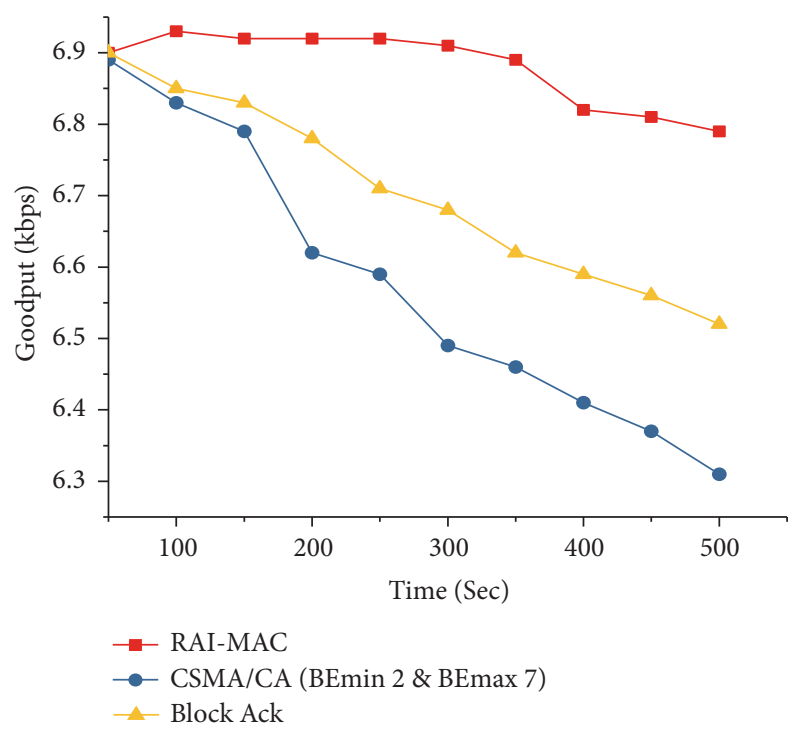

FIGURE 12: Average network goodput.

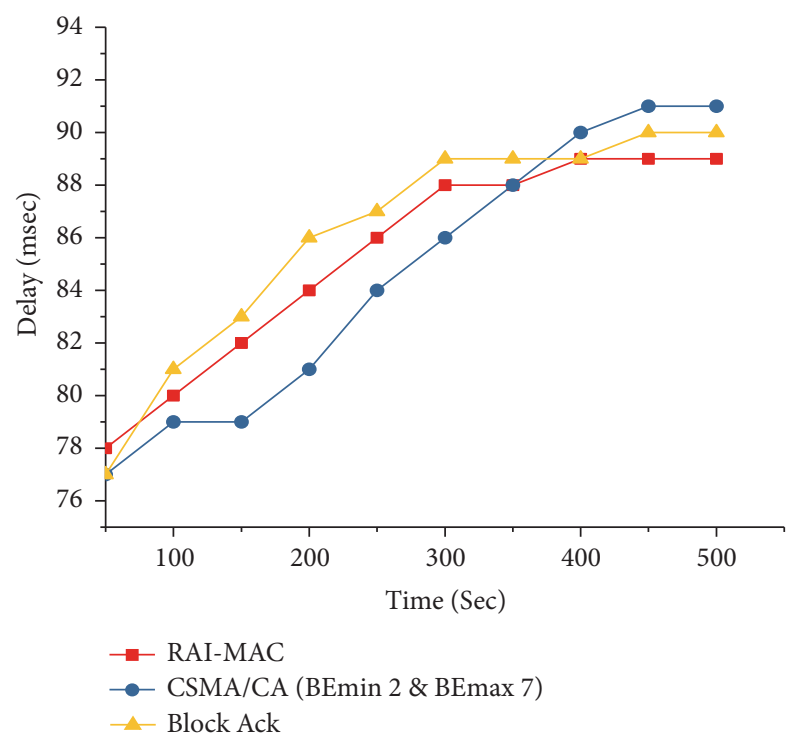

Figure 13: Average end-to-end delay.

of collisions. In Figure 13, end-to-end delay experienced by Block Ack is higher than RAI-MAC and CSMA (BEmin=2, BEmin=7). The Block Ack is based on TDMA in which any pending retransmissions are scheduled in the second phase of TDMA, thus incurring higher delay as compared to RAIMAC and CSMA (BEmin=2, BEmin=7).

Figure 14 shows the energy consumption comparison of RAI-MAC, CSMA (BEmin=2, BEmin=7), and Block Ack according to the ATMEL Mote transceiver. The results in this figure confirm that RAI-MAC scheme is as efficient in energy conservation as compared to the other two schemes. Our proposed scheme has almost similar energy performance trend as of CSMA (BEmin=2, BEmin=7). However, the Block Ack seems to have less energy consumption due to its well

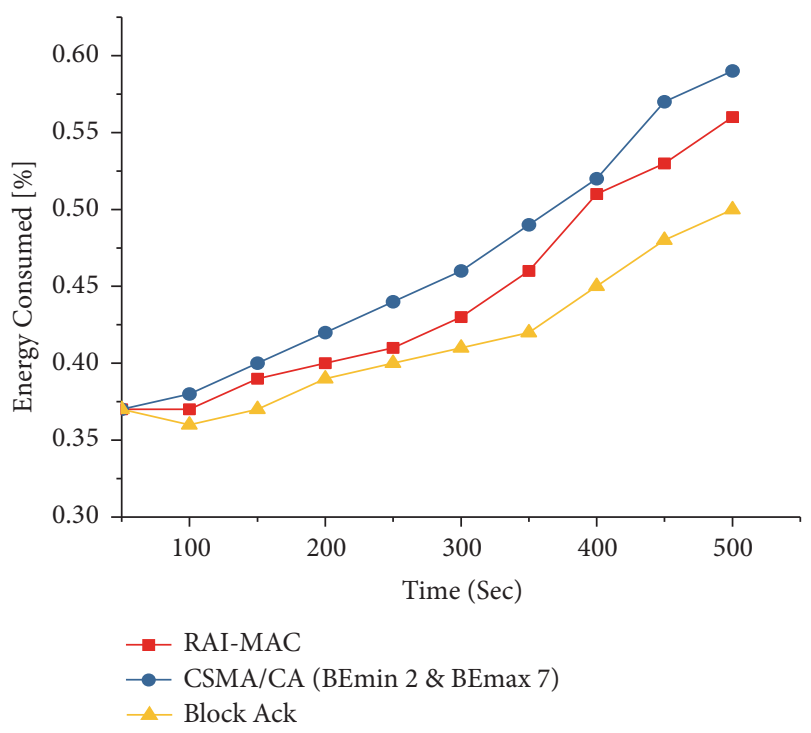

FIGURE 14: Average energy consumption.

scheduled retransmission mechanism as compared to CSMA $(\mathrm{BEmin}=2, \mathrm{BEmin}=7)$ and RAI-MAC.

7.3. Analysis of SO on RAI-MAC. The selection of an optimal $\mathrm{BO}$ and $\mathrm{SO}$ is one of the important tasks of the coordinator. In this section, we evaluate the performance of RAI-MAC under different $\mathrm{BO}$ and $\mathrm{SO}$. We run the simulations for a number of 15 sensor nodes with a coordinator and vary the traffic load from 0 to 3 packets/s assuming a packet size of 70 bytes. Other simulation parameters are the same as listed in Table 3.

As can be observed from Figure 15, lower SO values lead to lower average goodput. For lower values of SO, frequent beacon frames result in higher beacon overhead. Further, a lower value of SO requires frequent CCA attempts, resulting in higher collisions at the start of each superframe. As can be observed from the figure, this effect is more significant under high traffic loads.

Figure 16 shows that the end-to-end delay experienced by RAI-MAC increases significantly with an increase in traffic load. For the lower SO values, the probability of CCA deference by sensor nodes increases significantly resulting in lower end-to-end delay. However, for higher SO values, the RAI-MAC incurs higher backoff delays due to a decrease in CCA deference. It can be observed from Figure 17 that, for smaller SO values, RAI-MAC consumes more energy as compared to the larger values. This difference is more prominent for higher traffic loads.

\section{Conclusion}

In a WBAN, the exchange of information between sensor nodes and coordinator must be carried out in a reliable way to avoid loss of any important information. Although traditional CSMA/CA-based mechanism used by IEEE 802.15.4 is simple to provide access to the medium, however it lacks any retransmission ability which can increase the reliability 


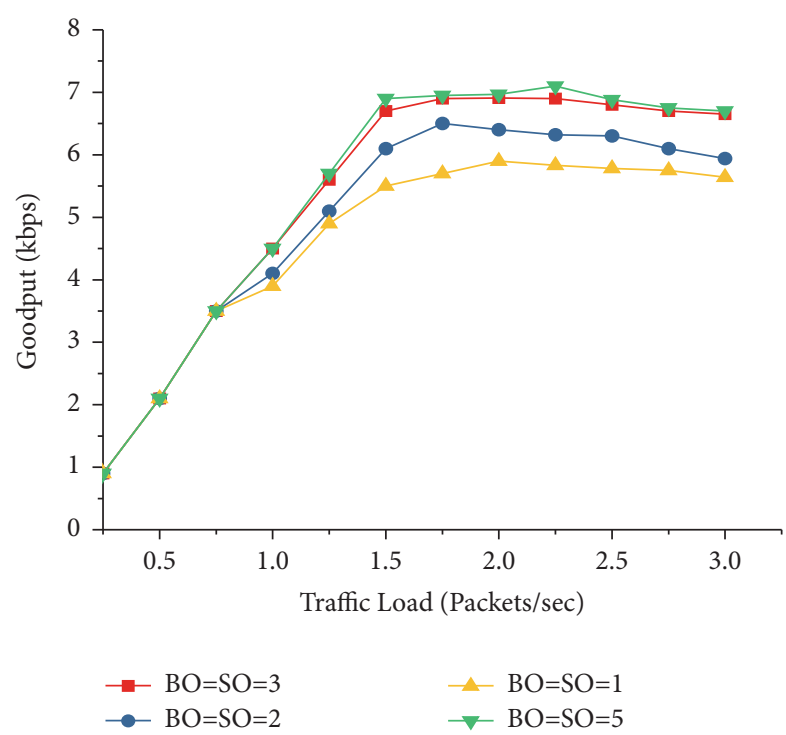

FIGURE 15: Goodput versus traffic load.

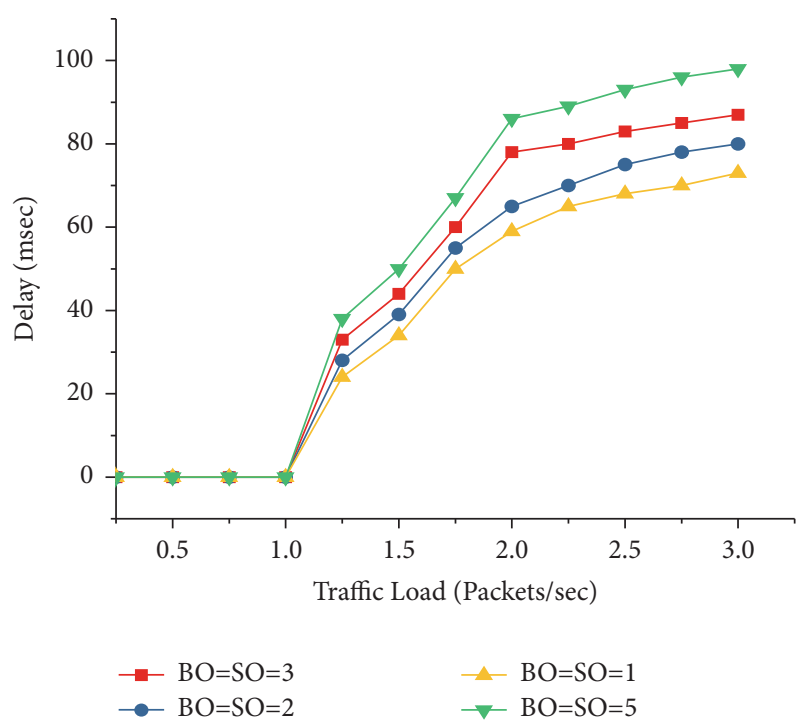

Figure 16: Average end-to-end delay versus traffic load.

of communication between sensor nodes and coordinator. There are several retransmission schemes which try to address the issues with the CSMA/CA; however these schemes do not offer any well-defined mechanism at the coordinator end, which can facilitate retransmissions according to the prevailing network conditions. This paper proposes a retransmission scheme, called RAI-MAC, which aims to improve the reliability of communication and to speed up the probability of successful transmissions in WBANs. When compared to CSMA/CA and other retransmission schemes, one of the most robust features of RAI-MAC is its dynamic adaptability to backoff time according to the number of failed transmissions as observed by the coordinator. One of the prime advantages of RAI-MAC is that coordinators classify,

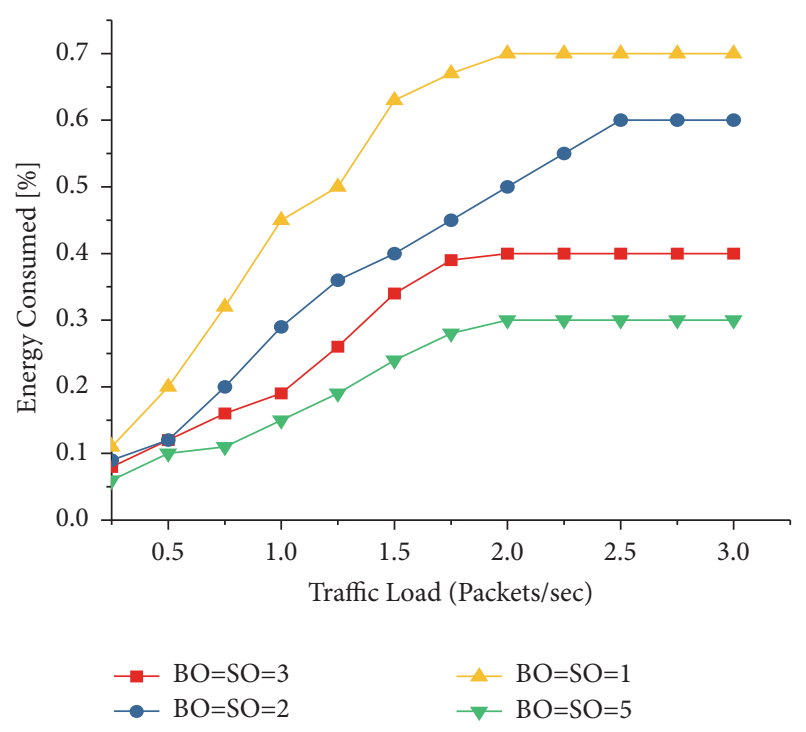

Figure 17: Average energy consumption versus traffic load.

prioritize, and control the retransmissions compared to other schemes, thereby improving the network performance.

Experimental assessments of RAI-MAC demonstrate that it has significant improved reliability behavior with low endto-end delay, while not compromising on energy efficiency when compared with the CSMA/CA and a popular TDMAbased retransmission scheme, i.e., Block Ack. Our proposed scheme improves the transmission reliability by considering the number of failed transmissions, however it does not consider the energy budget of each sensor node, independently. An important extension to our work can be based on retransmission classification and the remaining energy budget of each node. Further, it remains to be seen whether coordinator can make a better retransmission decision by using Q-learning approach [38].

\section{Data Availability}

Data sharing is not applicable to this article as no datasets were generated or analyzed during the current study.

\section{Conflicts of Interest}

The authors declare no conflicts of interest.

\section{Acknowledgments}

This work was supported by the Office of Research, Innovation and Commercialization (ORIC) at Bahria University, Islamabad.

\section{References}

[1] L. Luo, M. Zhang, J. Wei, W. Xu, Y. Zhang, and F. Shu, "Delay analysis of IEEE 802.15.4 slotted CSMA/CA considering deference mechanisms," in Proceedings of the International 
Conference on Wireless Communications and Signal Processing, WCSP 2015, October 2015.

[2] F. Hend, S. Khssibi, T. Val, H. Idoudi, A. V. den, and S. A. Leila, "Comparative study of IEEE 802.15.4 and IEEE 802.15.6 for WBAN-based CANet," in Proceedings of the 4th International Conference on Performance Evaluation and Modeling in Wireless Networks, pp. 1-7, 2016.

[3] D. De Guglielmo, F. Restuccia, G. Anastasi, M. Conti, and S. K. Das, "Accurate and efficient modeling of 802.15.4 unslotted CSMA/CA through event chains computation," IEEE Transactions on Mobile Computing, vol. 15, no. 12, pp. 2954-2968, 2016.

[4] H.-W. Tseng and Y.-R. Chuang, "A cross-layer judgment scheme for solving retransmission problem in ieee 802.15.4-based wireless body sensor networks," IEEE Sensors Journal, vol. 13, no. 8, pp. 3124-3135, 2013.

[5] H. Lee, A. Kim, K. Lee, and Y. Shin, "A improved channel access algorithm for IEEE 802.15.4 WPAN," International Journal of Security and Its Applications, vol. 6, no. 2, pp. 281-286, 2012.

[6] J. He, Z. Tang, H.-H. Chen, and S. Wang, "An accurate Markov model for slotted CSMA/CA algorithm in IEEE 802.15.4 networks," IEEE Communications Letters, vol. 12, no. 6, pp. 420422, 2008.

[7] R. Cavallari, F. Martelli, R. Rosini, C. Buratti, and R. Verdone, "A survey on wireless body area networks: technologies and design challenges," IEEE Communications Surveys \& Tutorials, vol. 16, no. 3, pp. 1635-1657, 2014.

[8] B. H. Jung, R. U. Akbar, and D. K. Sung, “Throughput, energy consumption, and energy efficiency of IEEE 802.15 .6 body area network (BAN) MAC protocol," in Proceedings of the 2012 IEEE 23rd International Symposium on Personal, Indoor and Mobile Radio Communications, PIMRC 2012, pp. 584-589, September 2012.

[9] S. Rashwand, J. Mišić, and H. Khazaei, "Performance analysis of IEEE 802.15.6 under saturation condition and error-prone channel," in Proceedings of the IEEE Wireless Communications and Networking Conference (WCNC '11), pp. 1167-1172, March 2011.

[10] S. Sarkar, S. Misra, B. Bandyopadhyay, C. Chakraborty, M. Obaidat, and S. Sarkar, "Performance analysis of IEEE 802.15.6 MAC protocol under non-ideal channel conditions and saturated traffic regime," IEEE Transactions on Computers, vol. 64, pp. 2912-2925, 2015.

[11] S. Ullah, M. Chen, and K. S. Kwak, "Throughput and delay analysis of IEEE 802.15.6-based CSMA/CA protocol," Journal of Medical Systems, vol. 36, no. 6, pp. 3875-3891, 2012.

[12] E. Sarra and T. Ezzedine, "Performance improvement of the wireless body area network (WBAN)," in Proceedings of the 18th IEEE International Conference on e-Health Networking, Applications and Services, Healthcom 2016, September 2016.

[13] X. Zhu, S. Han, P.-C. Huang, A. K. Mok, and D. Chen, "MBStar: a real-time communication protocol for wireless body area networks," in Proceedings of the 23rd Euromicro Conference on Real-Time Systems (ECRTS '11), pp. 57-66, July 2011.

[14] K. S. Deepak and A. V. Babu, "Enhancing reliability of IEEE 802.15.6 wireless body area networks in scheduled access mode and error prone channels," Wireless Personal Communications, vol. 89, no. 1, pp. 93-118, 2016.

[15] O. T. Valle, C. Montez, G. M. De Araujo, F. Vasques, and R. Moraes, "NetCoDer: a retransmission mechanism for WSNs based on cooperative relays and network coding," Sensors, vol. 16, no. 6, 2016.
[16] Y. Tselishchev, L. Libman, and A. Boulis, "Energy-efficient retransmission strategies under variable TDMA scheduling in body area networks," in Proceedings of the 36th Annual IEEE Conference on Local Computer Networks, LCN 2011, pp. 374-381, October 2011.

[17] D. Ghose and F. Y. Li, "Enabling retransmissions for achieving reliable multicast communications in WSNs", in Proceedings of the 23rd IEEE conference on vehicular technology, pp. 374-381, Bonn, Germany, 2011.

[18] W. Dong, J. Yu, and X. Liu, "CARE: corruption-aware retransmission with adaptive coding for the low-power wireless," in Proceedings of the 2015 IEEE 23rd International Conference on Network Protocols (ICNP), pp. 235-244, San Francisco, CA, USA, November 2015.

[19] H. Jeong, "An adaptive scheduling algorithm for the patient monitoring system on WBANs," in Proceedings of the Internet of Things: International Workshop, Changsha, China, 2012.

[20] B. Latré, B. Braem, I. Moerman, C. Blondia, and P. Demeester, "A survey on wireless body area networks," Wireless Networks, vol. 17, no. 1, pp. 1-18, 2011.

[21] Y. Tselishchev, L. Libman, and A. Boulis, "Reducing transmission losses in body area networks using variable TDMA scheduling," in Proceedings of the 2011 IEEE International Symposium on "A World of Wireless, Mobile and Multimedia Networks" (WoWMoM), pp. 1-10, Lucca, Italy, June 2011.

[22] G. Jianliang, H. Jia, and M. Geyong, "A new analytical model for slotted IEEE 802.15.4 medium access control protocol in sensor networks," in Proceedings of the 2009 WRI International Conference on Communications and Mobile Computing, CMC 2009, pp. 427-431, January 2009.

[23] K. Kunert, E. Uhlemann, and M. Jonsson, "Predictable real-time communications with improved reliability for IEEE 802.15.4 based industrial networks," in Proceedings of the 2010 8th IEEE International Workshop on Factory Communication Systems(WFCS 2010), pp. 13-22, Nancy, France, May 2010.

[24] G. Gamba, F. Tramarin, and A. Willig, "Retransmission strategies for cyclic polling over wireless channels in the presence of interference," IEEE Transactions on Industrial Informatics, vol. 6, no. 3, pp. 405-415, 2010.

[25] D. Nguyen, T. Tran, T. Nguyen, and B. Bose, "Wireless broadcast using network coding," IEEE Transactions on Vehicular Technology, vol. 58, no. 2, pp. 914-925, 2009.

[26] Q. Liu and A. Czylwik, "A collision-aware backoff mechanism for IEEE 802.15.4 wireless sensor networks," in Proceedings of the 6th IFIP/IEEE Wireless Days Conference, WD 2013, November 2013.

[27] S.-T. Sheu and Y.-Y. Shih, "A collision-free based rotational listening strategy (RLS) for IEEE 802.15.4 WPAN," in Proceedings of the 2007 IEEE International Conference on Communications, ICC'07, pp. 4535-4541, June 2007.

[28] A.-C. Pang and H.-W. Tseng, "Dynamic backoff for wireless personal networks," in Proceedings of the IEEE Global Telecommunications Conference, 2004. GLOBECOM '04, pp. 1580-1584, Dallas, TX, USA, 2004.

[29] A. Faridi, M. R. Palattella, A. Lozano et al., "Comprehensive evaluation of the IEEE 802.15.4 MAC layer performance with retransmissions," IEEE Transactions on Vehicular Technology, vol. 59, no. 8, pp. 3917-3932, 2010.

[30] E. Chatzistavros and G. Stamatelos, "Optimal Backoff Exponent estimation for environmental monitoring IEEE 802.15.4 startopology sensor networks," in Proceedings of the 2014 IEEE 
International Conference on Imaging Systems and Techniques, IST 2014, pp. 157-160, October 2014.

[31] K. Ashrafuzzaman and K. S. Kwak, "On the performance analysis of the contention access period of IEEE 802.15.4 MAC," IEEE Communications Letters, vol. 15, no. 9, pp. 986-988, 2011.

[32] A. N. Alvi, S. S. Naqvi, S. H. Bouk, N. Javaid, U. Qasim, and Z. A. Khan, "Evaluation of slotted CSMA/CA of IEEE 802.15.4," in Proceddings of the 7th International Conference on Broadband, Wireless Computing, Communication and Applications, BWCCA, pp. 391-396, 2012.

[33] S. Henna, M. Sajeel, F. Bashir, M. Asfand-E-Yar, and M. Tauqir, "A fair contention access scheme for low-priority traffic in wireless body area networks," Sensors, vol. 17, no. 9, pp. 914-925, 2017.

[34] J. Bhar, "A mac protocol implementation for wireless sensor network," Journal of Computer Networks and Communications, vol. 2015, 2015.

[35] J. Y. Ha, W. H. Kwon, T. H. Kim, S. Choi, and H. S. Park, "An enhanced CSMA-CA algorithm for IEEE 802.15.4 LR-WPANs," IEEE Communications Letters, vol. 11, no. 5, pp. 461-463, 2007.

[36] O. T. Valle, C. Montez, G. Arajo, P. Portugal, and F. Vasques, "Enhancing wireless sensor network simulators with a realistic battery discharge function," Information, vol. 16, pp. 8767-8780, 2013.

[37] ATMEL corporation, “802.15.4 MCUs," http://www.atmel.com/ products/wireless/802154/default.aspx, 2017.

[38] S. Galzarano, A. Liotta, and G. Fortino, "QL-MAC: a Q-learning based MAC for wireless sensor networks," in Proceedings of International Conference on Algorithms and Architectures for Parallel Processing, R. Aversa, J. Kołodziej, J. Zhang, F. Amato, and G. Fortino, Eds., pp. 267-275, 2013. 


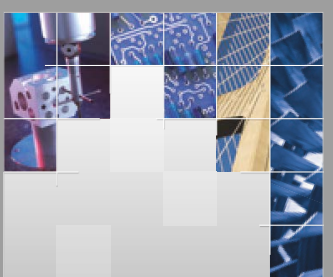

\section{Enfincering}
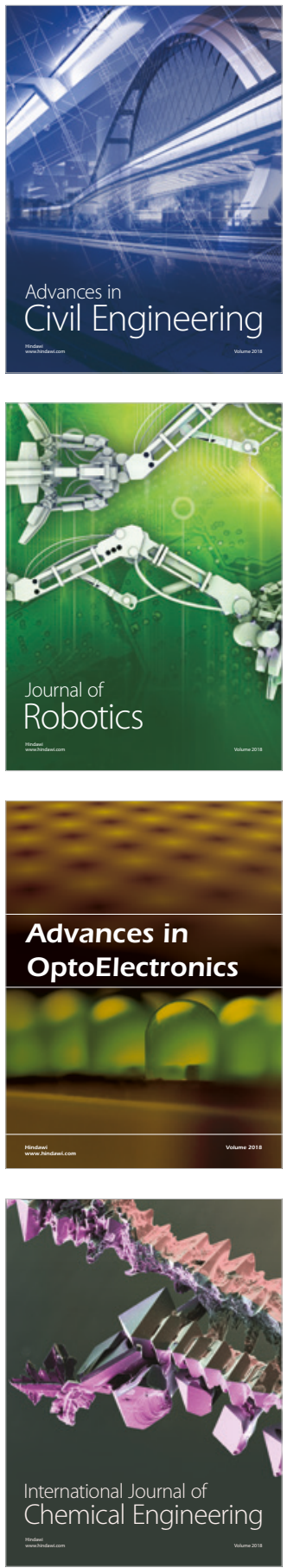

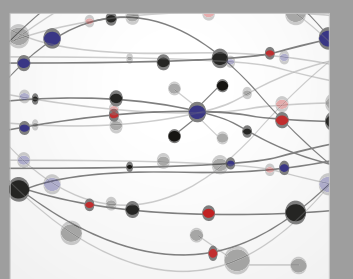

\section{Rotating \\ Machinery}

The Scientific World Journal

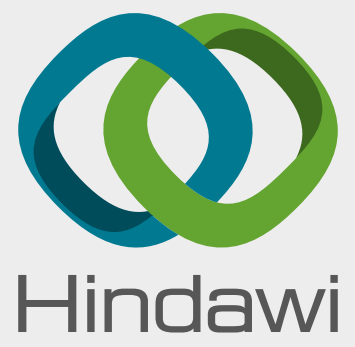

Submit your manuscripts at

www.hindawi.com
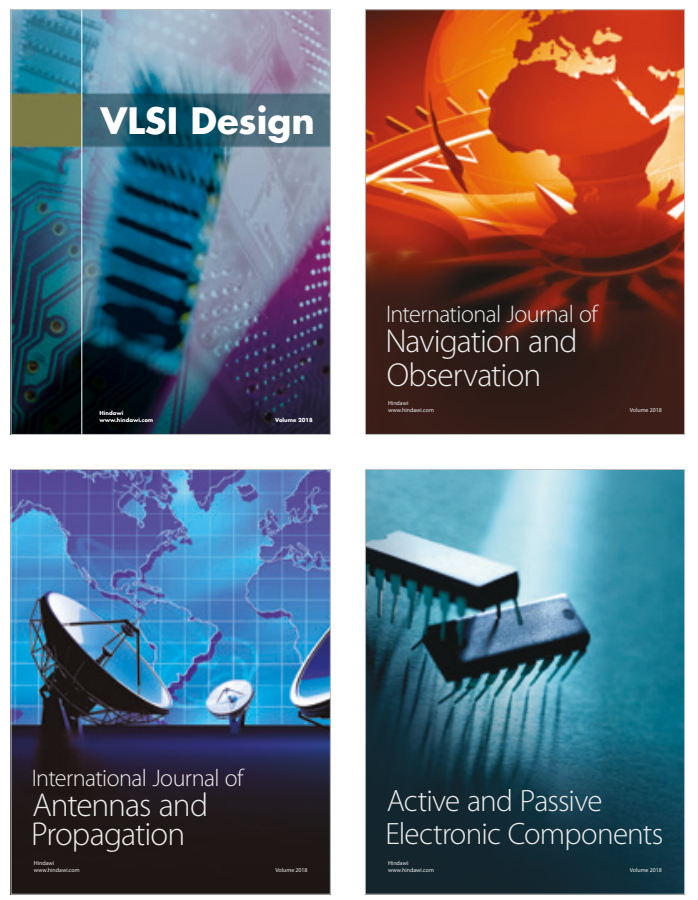
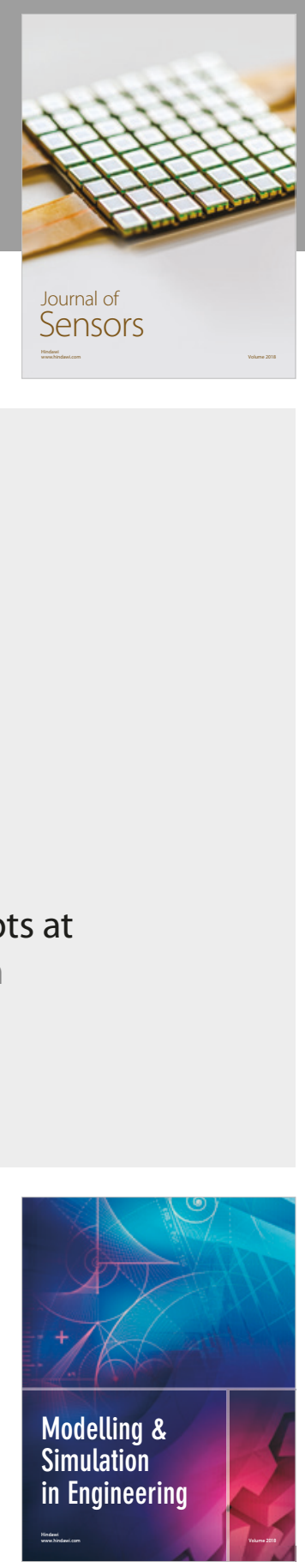

\section{Advances \\ Multimedia}
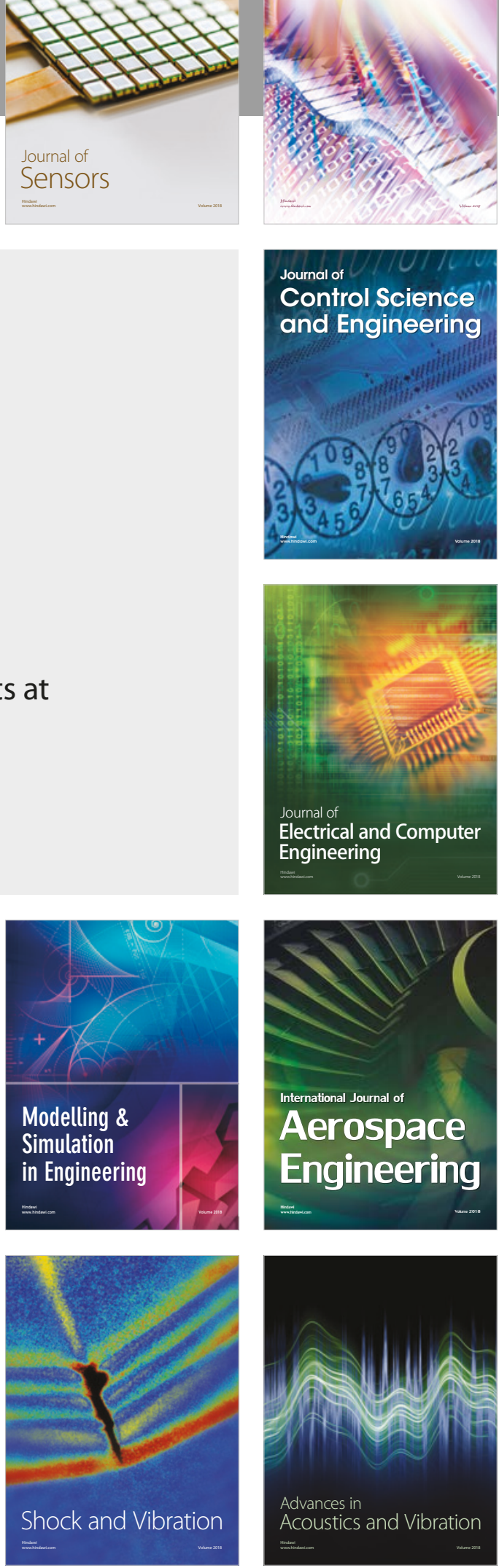